THURSDAY, FEBRUARY II, I886

\section{PRECAUTIONS AGAINST HYDROPHOBIA}

THE frequency of the terrible and incurable disease known as hydrophobia has led to precautions being adopted in London, which, if universally carried out, would probably rid us of the plague.

The recent progress of scientific pathology, i.e. of medical knowledge which advances by the methods of observation and experiment-has made clear much which was previously obscured by traditional fables.

It is now certain that hydrophobia is a real disease, not the mere result of fright, not primarily a mental disorder at all, but a definite sequence of bodily symptoms. Even its anatomy has been investigated by the beautiful methods of modern microscopical research ; and the coincidence of the results obtained by skilled histologists, working independently, makes it probable that the lesions discovered are essential and not accidental. If so, the anatomical part of the disease consists in a perivascular inflammation of the central nervous system, and particularly of the part known as the "bulb," or medulla oblongata, which is situated between the spinal marrow and the brain. Moreover, it has been ascertained that hydrophobia belongs to the group of "specific," or, as they were termed by the late Registrar-General, "zymotic," diseases, which always arise by contagion, and always "breed true." It is most improbable that it ever appears, either in man or animals, spontaneously or from common causes. In men and in brutes alike it follows the bite of a rabid dog, or other animal. How it first arose, and whether in dogs, wolves, foxes, cats, badgers, or in Carnivora before they were differentiated into the three groups of cats, dogs, and bears, with their several allied kinds--is quite unknown. But we have no historical knowledge of the origin of small-pox or measles, or even of diseases once thought to be unknown before modern times, such as syphilis, diphtheria, and cholera. The evolution of diseases, like that of the human beings and the brute creation they infest, is matter of speculation only.

Now there are three ways of dealing with these specific diseases. One is by treatment when they are fully developed. This is the business of the physician, and in many of them his treatment is so far effectual that, though prevention would be better than cure, yet cure is generally the result of rational treatment. But no effectual treatment of hydrophobia is known. In spite of the pretensions of charlatans and the constant attempts of physicians, no plan of treatment has yet been discovered which can show a single instance of success.

The second method is to arm the domain of life against an invading pest beforehand, instead of driving it out when already an entrance has been gained. This is the method first applied to small-pox by inoculation with imperfect success, and afterwards far more efficiently by Jenner's discovery of vaccination. No corresponding process of protection against other human diseases had been discovered until Pasteur's recent attempts to apply the principle to hydrophobia; but the same eminent savant VOL. XXXIII.-No. 850 bad before devised similar inoculations to prevent more than one epidemic disease peculiar to domestic animals. We referred in a previous number to his system of inoculation as a preservative against hydrophobia, and since then the cases on which he has operated have multiplied. One difficulty of judging as to the efficacy of his method is that not more than half the persons bitten by rabid dogs develop hydrophobia; the poisonous saliva may have been wiped off the teeth by the clothing as it was penetrated, or the effusion of blood may have immediately washed it away, or some local application may have destroyed it. Another is that hydrophobia has such an uncertain and often protracted period of "incubation," more uncertain and more protracted than that of any other specific disease, varying from a few weeks, or :ven possibly a few days, up to eleven or twelve months, and in some rare cases reaching two years, or possibly a longer period. But now that Pasteur's inoculations have considerably exceeded a hundred in number, these sources of fallacy are more likely to be eliminated, and as the mass of evidence increases, and the time grows longer, a conclusion one way or the other will become inevitable.

There is, however, a third method of dealing with hydrophobia, independent of future possible treatment and of inoculation. It is what the late Sir James Simpson called "stamping out" the infection in the case of cattleplague. If we could kill every rabid $\operatorname{dog}$ and wild animal throughout the world at once, we have reason to believe that hydrophobia would become of only historical interest. Happily it has never (so far as we know) been transmitted from one human being to another, so that it would not be necessary even to await the death of the victims already bitten before feeling secure. Destroy the disease in animals, and it would perish from among men.

But since the infection takes place in the great majority of cases by means of a dog's bite, it would be sufficient to prevent every rabid dog from biting. This of course is impossible: but if we could make a dog's bite a very rare instead of a very common occurrence, the chance of being bitten by a rabid dog would become indefinitely remote. If we could prevent dogs from biting one another, hydrophobia would cease from among dogs also. The rabid dogs would die innocuous. It has been proposed to draw the large canine teeth, but this would not entirely prevent dangerous bites, it could never be carried out thoroughly, it would give needless pain to intelligent animals, and in attempting to enforce it more bites would probably be inflicted on the operators than if the dogs had been let alone.

The only rational methods yet proposed of preventing, or rather of reducing, the number of dog-bites is first to diminish the number of dogs by imposing a higher tax on those kept as domestic animals, and by destroying ownerless, miserable, and.half-starved curs ; and secondly to prevent dogs biting when abroad by enforcing the use of muzzles. These may be constructed so as not to interfere with the animal breathing, perspiring, and even drinking with comfort, and yet to prevent his using his teeth.

Such muzzles are enforced and worn by a recent regulation of the Metropolitan Police, and troublesome as such interference with individual liberty (whether of dogs or their masters) is felt to be in this country, it may be 
hoped that the rational part of the community will see its reasonableness, and will do their best to have it thoroughly carried out.

Similar measures have long been enforced in Berlin and other Continental cities, and have been followed by the most gratifying results in the diminution or suppression of the dreaded hydrophobia.

\section{BOTANICAL RESULTS OF THE "CHAL-} LENGER" EXPEDITION

Report on the Sicntific Results of the Voyage of H.M.S. "Challenger" during the Years 1873-76 under the Command of Capt. G. S. Nares, R.N., F.R.S., and Capt. F. T. Thomson, R.N. Prepared under the Superintendence of the late Sir C. Wyville Thomson, F.R.S., \&c., and now of John Murray, one of the Naturalists of the Expedition. Botany-Vol. I. By William Botting Hemsley, A.L.S. (Published by Order of Her Majesty's Government, I 885.)

THE botanical results of the voyage of the Cliallenger hitherto published have been confined to the reports of Mr. Moseley, sent home from time to time during the voyage, along with collections of plants made by him, to Kew, and published, along with lists of the species, in the Fournal of the Linnean Society. In the volume before us we have the results of a more detailed working up of the material thus obtained so far as it belongs to insular floras. Mr. Hemsley, who is responsible for the book, brings to his task botanical experience which begets the liveliest confidence in the thoroughness and accuracy of the work, and the volume will certainly compare with any of those upon the zoological results of the voyage already published. It is necessary to emphasise the fact that Mr. Hemsley is the author of the book, for it is not patent on first inspection. The title-page, where one naturally looks for information upon the subject of authorship, tells that the volume is published under the superintendence of Mr. John Murray, who writes barely a page of preface, but it says nothing of Mr. Hemsley, the author of the rest of the book-over 900 pages-and it is only by turning up the table of contents that his connection with the book is found recorded. This may be consistent with uniformity in the appearance of the Challenger publications, but hardly, we think, with consideration for the author.

Although appearing amongst the scientific results of the Challenger voyage, Mr. Hemsley's work has a considerably wider basis than the botanical insular collections made during that voyage. From the rich stores of the herbaria at Kew and the British Museum he has sought out and made use of collections and records of observations of travellers, both old and recent, so far as they relate to islands from which Mr. Moseley procured specimens, and he is thus enabled to present an account of all that is known of the vegetation of these islands,-in some cases their complete flora. The book is, then, no mere descriptive synopsis of the botany of the Challenger Expedition. Its nature may be gathered from Mr. Hemsley's own description :-

"In the introductory notes on the vegetation of the various islands included in the reports on the botany of the Expedition are embodied tables showing the distribution of the genera and species of each island or group of islands. There are also observations on the composition, affinities, \&c., of the different insular floras, together with references to the diverse agencies operating in the dispersal of plants, whilst numerous facts and suggestions bearing upon the same subject are scattered throughout the lists. Finally, the appendix to the third part of the botany is devoted to the record of evidence of the part played by oceanic currents and birds in the transport of seeds from place to place. The general introduction is not limited to a mere summary of the facts contained in the reports, and speculations thereon; it has been so extended as to form an epitome of the botany of a large number of oceanic islands and of the Antarctic regions generally. The special characteristics of insular vegetation in various parts of the world are set forth and compared with continental vegetation; and, as a whole, the work may perhaps serve as an indication to travellers of the nature and extent of the observations required for the advancement of this most interesting subject."

As to method the book is divided into four parts. The first is a "Report on the Present State of Knowledge of various Insular Floras," being an introduction to the botany of the Challenger Expedition. The second and third parts are Reports "On the Botany of the Bermudas and various other Islands of the Atlantic and Southern Oceans," namely, St. Paul's Rocks, Fernando Noronha, Ascension, St. Helena, South Trinidad, Tristan d'Acunha Group, and St. Paul and Amsterdam Islands, and the chain of islands from the Prince Edward Group to the Macdonald Group. The fourth part consists of a "Report on the Botany of Juan Fernandez with Masafuera, San Ambrosio, and San Felix, the South-Eastern Moluccas, and the Admiralty Islands," to which is added an Appendix "On the Dispersal of Plants by Oceanic Currents and Birds."

The scope of the volume is so comprehensive, and the subject is in touch with so many of the interesting problems connected with plant-distribution, that in this notice we must content ourselves with merely indicating some of the more prominent features that characterise the book. At the outset we may state that it abounds with interesting and new facts, and the analytical tables graphically representing various facts of distribution, compiled evidently with great care, are exceedingly striking, and bring out contrasts in a manner no amount of writing could effect.

The introductory part is really a series of essays upon variousinteresting subjects connected with phytogeography, which are mainly treated in their bearing on insular fioras. An early chapter deals with the classification of islands in relation to the composition of their vegetation. Wallace's classification of oceanic and continental islands, whilst harmonising with the features of vegetation of many islands, is not adapted, Mr. Hemsley thinks, for exhibiting the floral peculiarities of islands generally. The Bermudas and Galapagos, for example, included in Wallace's oceanic group, cannot be fairly placed in the same category. He therefore proposes a classification which in effect, so far as it is applied in this volume, amounts to the subdividing of Wallace's oceanic group into ancient, more recent, and new sub-groups. Islands may be arranged, he says, for phytogeographic purposes, in three categories, according to their endemic element, 